\title{
Optical detection of a Bardeen-Cooper-Schrieffer phase transition in a trapped gas of fermionic atoms
}

\author{
Weiping Zhang, ${ }^{1,2}$ C. A. Sackett, ${ }^{2}$ and R. G. Hulet ${ }^{2}$ \\ ${ }^{1}$ School of Mathematics, Physics, Computing and Electronics, Macquarie University, Sydney, New South Wales 2109, Australia \\ ${ }^{2}$ Physics Department and Rice Quantum Institute, Rice University, Houston, Texas 77251
}

(Received 6 October 1998)

\begin{abstract}
A theoretical treatment of light scattering from a degenerate Fermi gas of trapped ultracold ${ }^{6} \mathrm{Li}$ atoms is presented. We find that the scattered light contains information that directly reflects the quantum pair correlation due to the formation of atomic Cooper pairs resulting from a Bardeen-Cooper-Schrieffer phase transition to a superfluid state. Evidence for pairing should be observable in both the space and time domains. [S1050-2947(99)09207-0]
\end{abstract}

PACS number(s): 03.75.Fi, 32.80.Pj, 67.40.Db

The realization of Bose-Einstein condensation (BEC) in trapped atomic gases [1] has generated interest in the atomic physics, quantum optics, and condensed-matter physics communities. Although the experimental realization of a degenerate atomic Fermi gas has not yet been demonstrated, interest in this subject is also increasing [2-6], and efforts to trap and cool ${ }^{6} \mathrm{Li}$ and ${ }^{40} \mathrm{~K}$ gases into the quantum degenerate regime are underway in several laboratories. Of course, the behavior of a degenerate Fermi gas is remarkably different from that of a degenerate Bose gas. As in the BardeenCooper-Schrieffer (BCS) theory of superconductivity in metals, it has been predicted that a degenerate Fermi gas can undergo a BCS phase transition if the interatomic interactions in the gas are attractive [7]. In this phenomenon, the Fermi gas is cooled to near absolute zero, so that all trap levels up to the Fermi energy are filled. Attractive interactions can then cause atoms in the vicinity of the Fermi level to form Cooper pairs, with each pair composed of two quantum correlated atoms behaving as a composite Bose particle. In the BCS theory, these bosons automatically undergo BEC and form an atomic superfluid, with the quantum pair correlation of the Cooper pairs characterizing the superfluid properties of the gas. In this paper, we address the question of how to detect this superfluid state once the transition has occured.

A promising experimental system consists of a degenerate gas with atoms in an incoherent mixture of two internal hyperfine states. Such a mixture allows Cooper pairing via an $s$-wave interaction, and leads to practically attainable BCStransition temperatures when the scattering length $a$ is large and negative. This occurs naturally for ${ }^{6} \mathrm{Li}[8]$, or can be obtained in the vicinity of a Feshbach resonance for other atoms [9]. We consider here a trapped ${ }^{6} \mathrm{Li}$ gas in an incoherent mixture of ground states $|+\rangle=\left|M_{s}=1 / 2, M_{I}=1\right\rangle$ and $|-\rangle=\left|M_{s}=1 / 2, M_{I}=0\right\rangle[2]$.

The key to observing the superfluid state is to determine the existence of pair correlations. To achieve this goal, we propose to use off-resonance light scattering and Fourierimaging techniques. A laser beam with amplitude $\mathbf{E}_{L}$, frequency $\omega_{L}$, and wave-vector $k$ propagating along the $z$ direction is used to illuminate the gas. We take the light to be linearly polarized and tuned near resonance between the $2 S$ ground state and $2 P$. excited state. To avoid incoherent heating of the gas due to spontaneous emission, the magnitude of the laser detuning, $\delta=\omega_{L}-\omega_{0}$, is assumed to be large compared to the transition linewidth $\gamma$. In vector quantum-field theory [10-12], the atoms in the light field can be described by a four-component atomic field $\Psi(\mathbf{r})=\psi_{+}|+\rangle+\psi_{-}|-\rangle$ $+\psi_{e+}|e+\rangle+\psi_{e-}|e-\rangle$ with $\psi_{ \pm}$denoting atoms in the ground-state hyperfine levels $| \pm\rangle$, and $\psi_{e \pm}$ in the corresponding excited-state hyperfine levels. For large $\delta$, the excited-state components can be adiabatically eliminated, yielding a total atomic polarization operator with positivefrequency part [10]

$$
\mathbf{P}^{(+)}(\mathbf{r}, t)=-\wp \frac{\wp \cdot \mathbf{E}^{(+)}}{\hbar \delta} \hat{\rho}(\mathbf{r}, t) e^{-i \omega_{L} t},
$$

where $\hat{\rho}(\mathbf{r}, t)=\psi_{+}^{\dagger}(\mathbf{r}, t) \psi_{+}(\mathbf{r}, t)+\psi_{-}^{\dagger}(\mathbf{r}, t) \psi_{-}(\mathbf{r}, t)$ is the total atomic density operator in the ground state, $\wp$ is the matrix element of the atomic dipole moment, and $\mathbf{r}$ is a location in the gas. Light propagation is determined by the atomic polarization operator (1) and the wave equation

$$
\nabla^{2} \mathbf{E}^{(+)}-\frac{1}{c^{2}} \frac{\partial^{2} \mathbf{E}^{(+)}}{\partial t^{2}}=\mu_{0} \frac{\partial^{2} \mathbf{P}^{(+)}}{\partial t^{2}} .
$$

The solution to Eq. (2) can be expressed as

$$
\mathbf{E}^{(+)}(\mathbf{R}, t)=\mathbf{E}_{S}^{(+)}(\mathbf{R}, t) e^{-i \omega_{L} t}+\mathbf{E}_{L}^{(+)} e^{i k z-i \omega_{L} t},
$$

where $\mathbf{E}_{S}^{(+)}(\mathbf{R}, t)$ is the scattered field at position $\mathbf{R}$. For $R$ $\equiv|\mathbf{R}| \gg|\mathbf{r}|$, the scattered field has the form $[10,12]$,

$$
\begin{aligned}
\mathbf{E}_{S}^{(+)}(\mathbf{R}, t)= & k^{2} \frac{e^{i k R}}{R} \int d^{3} r e^{-i k \hat{\mathbf{R}} \cdot \mathbf{r}} \\
& \times\left[\mathbf{P}^{(+)}(\mathbf{r}, t)-\hat{\mathbf{R}} \cdot \mathbf{P}^{(+)}(\mathbf{r}, t) \hat{\mathbf{R}}\right],
\end{aligned}
$$

where the directional unit vector $\hat{\mathbf{R}}=\mathbf{R} / R$. From Eqs. (1) and (4), we see that the scattered field depends on the density operator of the gas, so that the averaged spectral intensity of the scattered field received by a photodetector contains the second-order correlation of the atomic field operators [14], 


$$
\left\langle\hat{\rho}(\mathbf{r}, t) \hat{\rho}\left(\mathbf{r}^{\prime}, t^{\prime}\right)\right\rangle \approx\langle\hat{\rho}(\mathbf{r}, t)\rangle\left\langle\hat{\rho}\left(\mathbf{r}^{\prime}, t^{\prime}\right)\right\rangle+G\left(\mathbf{r}, \mathbf{r}^{\prime}, t, t^{\prime}\right),
$$

where $\langle\cdots\rangle$ denotes the quantum-mechanical expectation value. The first term in Eq. (5), which depends on the total averaged density, describes the contribution to the scattered field by the normal ground-state component. The second term,

$$
G\left(\mathbf{r}, \mathbf{r}^{\prime}, t, t^{\prime}\right) \equiv-2\left\langle\psi_{-}(\mathbf{r}, t) \psi_{+}\left(\mathbf{r}^{\prime}, t^{\prime}\right)\right\rangle\left\langle\psi_{-}^{\dagger}(\mathbf{r}, t) \psi_{+}^{\dagger}\left(\mathbf{r}^{\prime}, t^{\prime}\right)\right\rangle,
$$

gives the quantum pair correlation function arising from the formation of Cooper pairs in the superfluid state.

The contribution of the laser field $\mathbf{E}_{L}$ in Eq. (3) can be removed by imaging the cloud with a dark ground technique, as discussed in Refs. [13]. If a plane located a distance $z_{0}$ from the atoms is observed in this way, the spectral and spatial intensity distribution measured on the detector will be [14]

$$
\begin{aligned}
I\left(\mathbf{R}_{\perp}, \nu\right)= & \int_{-\infty}^{\infty} d \tau e^{i \nu \tau} \frac{1}{2 T} \int_{-T}^{T} d t\left\langle\mathbf{E}_{S}^{(-)}\left(\mathbf{R}_{0}, t\right)\right. \\
& \left.\times \mathbf{E}_{S}^{(+)}\left(\mathbf{R}_{0}, t+\tau\right)\right\rangle,
\end{aligned}
$$

where $2 T$ is the time interval used for detection, and $\mathbf{R}_{0}$ $\equiv\left(\mathbf{R}_{\perp}, z_{0}\right)$ is a point in the image plane. Equation (4), along with relations (1) and (5), gives the spatial-temporal correlation function of the light field,

$$
\begin{aligned}
\left\langle\mathbf{E}_{S}^{(-)}\left(\mathbf{R}_{0}, t\right) \cdot \mathbf{E}_{S}^{(+)}\left(\mathbf{R}_{0}, t+\tau\right)\right\rangle & \\
\quad= & \frac{9 I_{L} \gamma^{2}}{16\left(k z_{0} \delta\right)^{2}}\left[I_{1}\left(\mathbf{R}_{\perp}, t, \tau\right)+I_{2}\left(\mathbf{R}_{\perp}, t, \tau\right)\right] e^{-i \omega_{L} \tau},
\end{aligned}
$$

where $I_{L}=\mathbf{E}_{L}^{(-)} \cdot \mathbf{E}_{L}^{(+)}$is the intensity of the incident light. The functions $I_{1}$ and $I_{2}$ are defined as

$$
\begin{aligned}
I_{1}\left(\mathbf{R}_{\perp}, t, \tau\right)= & \iint d^{2} \mathbf{r}_{\perp} d^{2} \mathbf{r}_{\perp}^{\prime} e^{-i k \mathbf{R}_{\perp} \cdot\left(\mathbf{r}_{\perp}-\mathbf{r}_{\perp}^{\prime}\right) / z_{0}} \\
& \times\left\langle\hat{\rho}\left(\mathbf{r}_{\perp}, t\right)\right\rangle\left\langle\hat{\rho}\left(\mathbf{r}_{\perp}^{\prime}, t+\tau\right)\right\rangle,
\end{aligned}
$$

and

$$
I_{2}\left(\mathbf{R}_{\perp}, t, \tau\right)=\int d^{2} \xi e^{-i k \mathbf{R}_{\perp} \cdot \xi / z_{0}} \int d^{2} \mathbf{r}_{\perp} G\left(\mathbf{r}_{\perp}, \mathbf{r}_{\perp}-\boldsymbol{\xi}, t, t+\tau\right),
$$

where the relative distance between atoms is denoted by $\boldsymbol{\xi}$ $=\mathbf{r}_{\perp}-\mathbf{r}_{\perp}^{\prime}$. The function $I_{1}$ describes the signal from the normal component of the gas and $I_{2}$ describes the signal from the Cooper pairs. In general, $I_{2}$ is much weaker than $I_{1}$ since the averaged density of atoms in the normal component is far larger than that of the pairs.

The averaged density and the quantum pair-correlation function can be found using vector quantum-field theory [10]. In the off-resonant light field, the degenerate Fermi gas is described by the coupled quantum-field equations,

$$
\begin{gathered}
i \hbar \frac{\partial \psi_{+}}{\partial t}=\left(H_{0}-\mu_{+}+V_{L}-i \hbar \Gamma / 2\right) \psi_{+}-\Delta(\mathbf{r}) \psi_{-}^{\dagger}, \\
i \hbar \frac{\partial \psi_{-}^{\dagger}}{\partial t}=-\left(H_{0}-\mu_{-}+V_{L}+i \hbar \Gamma / 2\right) \psi_{-}^{\dagger}-\Delta(\mathbf{r}) \psi_{+},
\end{gathered}
$$

where $H_{0}=-\left(\hbar^{2} / 2 m\right) \nabla^{2}+\frac{1}{2} m \omega^{2} r^{2}$ is the free Hamiltonian of the trapped Fermi gas, $V_{L}=\hbar \Omega^{2} / 4 \delta$ is the light-induced potential, $\Gamma=\gamma \Omega^{2} / 4 \delta^{2}$ is the rate for spontaneous emission, $\mu_{ \pm}$are the chemical potentials of the two internal states, and $\Delta(\mathbf{r})=\left(4 \pi|a| \hbar^{2} / m\right)\left\langle\psi_{-}(\mathbf{r}) \psi_{+}(\mathbf{r})\right\rangle$ is the BCS energy-gap function [2]. The Rabi frequency of the light field is $\Omega$ $\equiv\left|\wp \cdot \mathbf{E}_{L} / \hbar\right|$. For simplicity we consider the case $\mu_{+}=\mu_{-}$ with equal numbers of atoms in each spin state, and introduce the renormalized chemical potential $\mu=\mu_{+}-V_{L}$. Further, a large laser detuning and a weak intensity allow $\Gamma$ $\ll \mu, \Delta$ so that destruction of Cooper pairs by spontaneous emission and interactions involving excited-state atoms [10] can be neglected. Employing an approach similar to that adopted in BCS theory, we approximate the solutions of Eqs. (11) by

$$
\psi_{ \pm}(\mathbf{r}, t)=\sum_{\mathbf{n}}\left[u_{\mathbf{n}}(\mathbf{r}) \hat{b}_{\mathbf{n} \pm} e^{-i E_{\mathbf{n}}^{t / \hbar}} \pm v_{\mathbf{n}}(\mathbf{r}) \hat{b}_{\mathbf{n}+}^{\dagger} e^{i E_{\mathbf{n}} t / \hbar}\right]
$$

where $\hat{b}_{\mathbf{n} \pm}$ are generalized Bogoliubov quasiparticle operators and $E_{\mathbf{n}}$ is the excitation energy for the mode indexed by n. The superfluid state of the degenerate Fermi gas is characterized by the BCS ground state $\left|\Phi_{B C S}\right\rangle$ with the property $\hat{b}_{\mathbf{n} \pm}\left|\Phi_{B C S}\right\rangle=0$. From Eqs. (11) with the dissipative terms ignored, the transformation coefficients $\left\{u_{\mathbf{n}}, v_{\mathbf{n}}\right\}$ satisfy the celebrated Bogoliubov equations,

$$
\begin{gathered}
\left(H_{0}-\mu\right) u_{\mathbf{n}}(\mathbf{r})+\Delta(\mathbf{r}) v_{\mathbf{n}}(\mathbf{r})=E_{\mathbf{n}} u_{\mathbf{n}}(\mathbf{r}), \\
-\left(H_{0}-\mu\right) v_{\mathbf{n}}(\mathbf{r})+\Delta(\mathbf{r}) u_{\mathbf{n}}(\mathbf{r})=E_{\mathbf{n}} v_{\mathbf{n}}(\mathbf{r}) .
\end{gathered}
$$

The total averaged density can be expressed as $\langle\hat{\rho}(\mathbf{r}, t)\rangle$ $\equiv\left\langle\Phi_{B C S}\left|\psi_{+}^{\dagger} \psi_{+}+\psi_{-}^{\dagger} \psi_{-}\right| \Phi_{B C S}\right\rangle=2 \Sigma_{\mathbf{n}}\left|v_{\mathbf{n}}(\mathbf{r})\right|^{2}$ and the quantum pair function is

$$
\begin{aligned}
G\left(\mathbf{r}, \mathbf{r}^{\prime}, t, t^{\prime}\right)= & 2 \sum_{\mathbf{n m}} u_{\mathbf{n}}(\mathbf{r}) v_{\mathbf{n}}\left(\mathbf{r}^{\prime}\right) u_{\mathbf{m}}\left(\mathbf{r}^{\prime}\right) v_{\mathbf{m}}(\mathbf{r}) \\
& \times e^{-i\left(E_{\mathbf{n}}+E_{\mathbf{m}}\right)\left(t-t^{\prime}\right) / \hbar}
\end{aligned}
$$

The average density and pair function can be calculated by self-consistently solving Eqs. (13). In the normal degenerate ground state, energy levels below the Fermi level $E_{F}$ are occupied, while those above are empty. The effect of interatomic interactions is to cause scattering between nearby energy levels, and creates an energy shell near $E_{F}$ where normally unoccupied states acquire an amplitude to be occupied, and states below $E_{F}$ have some amplitude to be unoccupied. The stronger the interatomic interaction is, the wider the energy shell and the more atoms are available to form Cooper pairs. Physically, the coefficients $u_{\mathbf{n}}$ and $v_{\mathbf{n}}$ in Eqs. (13) determine the amplitudes for atoms to be scattered into the pair states. To evaluate these amplitudes, we expand the 


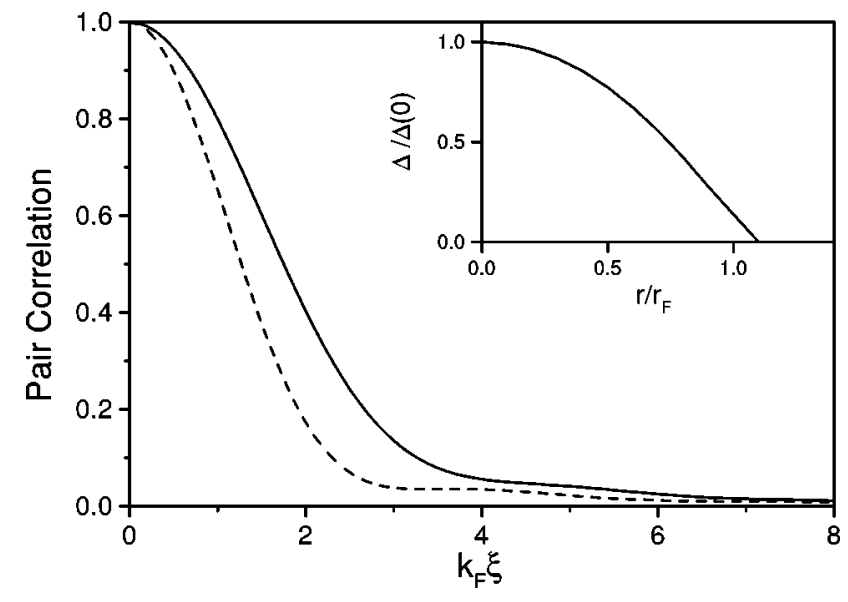

FIG. 1. The normalized spatial distributions of the equal-time quantum pair-correlation function, with the distance $\xi$ between atoms scaled by the Fermi wave number $k_{F}$. The dashed curve is the correlation due to Cooper pairs with center of mass located at the trap center and the solid curve is the average contribution of all Cooper pairs. The inset shows the spatial dependence of the energy gap, normalized to the center of the trap and with position scaled by the Fermi radius $r_{F}$.

coefficients as $u_{\mathbf{n}}=\Sigma_{\mathbf{q}} u_{\mathbf{n} \mathbf{q}} \phi_{\mathbf{q}}$ and $v_{\mathbf{n}}=\Sigma_{\mathbf{q}} v_{\mathbf{n q}} \phi_{\mathbf{q}}$, in terms of the eigenstates $\phi_{\mathbf{q}}$ of the single-atom Hamiltonian $H_{0}$. In principle, the sum over $\mathbf{q}$ in the coefficients should extend from zero to infinity. However, in the BCS theory [2,3], Eq. (13) is derived using the Born approximation, by replacing the nonlocal interatomic interaction $V(\mathbf{r})$ by a local contact potential $V(\mathbf{r})=4 \pi \hbar^{2} a \delta(\mathbf{r}) / m$. However, the Born approximation is only valid for low-energy scattering, and the inaccuracy of the approximation in the high-energy regime is known to cause an ultraviolet (UV) divergence. In the case of superconductivity, the UV divergence is naturally removed by considering the fact that the phonon-exchangeinduced interaction between electrons must cut off at the Debye frequency. However, in the case of an atomic gas, avoiding the UV divergence requires a more exact theory accounting for the real shape of $V(\mathbf{r})$. Two approaches to this problem have recently been proposed. One is to renormalize the interaction potential in terms of the LippmanSchwinger equation [2], and this is the approach we have taken here, and the other is to employ a more sophisticated pseudopotential approximation [3]. For the Born approximation to be valid, the cutoff momentum $\hbar k_{c}$ must satisfy $k_{c}$ $<|a|^{-1}$. The other momentum scale present is the Fermi momentum $\hbar k_{F}=\left(2 m E_{F}\right)^{1 / 2}$, which for ${ }^{6} \mathrm{Li}$ under reasonable experimental conditions is comparable to $|a|^{-1}$. As a check on the renormalization procedure, we find that values obtained for both $G$ and $\Delta(0)$ are hardly affected by changing $k_{c}$ from $|a|^{-1}$ to $|2 a|^{-1}$.

To be concrete, we assume that $N=2 \times 10^{5}{ }^{6} \mathrm{Li}$ atoms in each spin state are confined in a magnetic trap with an oscillation frequency $\omega=2 \pi \times 150 \mathrm{~Hz}$. With these values, $E_{F}$ $\approx 100 \hbar \omega \approx 740 \mathrm{nK}$, and the peak value of the energy gap is $\Delta(0) \approx 5 \hbar \omega=36 \mathrm{nK}$. For a degenerate Fermi gas in a harmonic trap, the characteristic size of the average density is given by the Fermi radius $r_{F}=\left(2 E_{F} / m \omega^{2}\right)^{1 / 2} \approx 48 \mu \mathrm{m}$, while the length scale of the pair-correlation function $G$ is $r_{c} \sim k_{F}^{-1} \approx 0.23 \mu \mathrm{m}$. The numerical result for the correlation
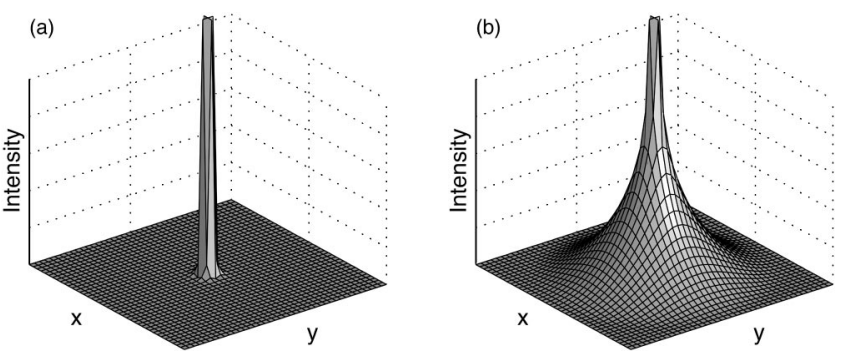

FIG. 2. The spatial image measured a distance $z_{0}$ from the atoms, with (a) the trapped Fermi gas in the normal degenerate ground state and (b) in the superfluid state after the BCS phase transition. In both images, the central peak is clipped and actually extends by a factor of $\sim 10^{6}$ above the axes shown. The radial size of the normal component is approximately $z_{0} / k r_{F}$, while the pair component is larger, extending to $z_{0} / k r_{c}$.

function is shown in Fig. 1, along with the spatial variation of the energy gap.

In studies of a homogeneous gas, the correlation length at zero temperature is conventionally expressed in terms of the coherence length $\xi_{c}=2 k_{F}^{-1} E_{F} / \Delta(0)$, which determines the region over which the pair function extends [6]. Within this region, however, the pair function oscillates on the shorter length scale $r_{c}$. The numerical results for the trapped gas in Fig. 1 similarly show that the pair function varies substantially across the distance $k_{F}^{-1}$, while the tail of the function does indeed extend to approximately $\xi_{c}$.

It is the short-length-scale variations that allow the pair function to be optically detected. If a plane at $z_{0}=2 \mathrm{~cm}$ is imaged with unit magnification and with a transition wavelength $\lambda=670 \mathrm{~nm}$, the image size will be $z_{0} / k r_{F}$ $\sim 0.09 \mathrm{~mm}$ for the normal component and $z_{0} / k r_{c} \sim 1.9 \mathrm{~cm}$ for the pair component, differing by a factor of $2 E_{F} / \hbar \omega$. The calculated images for a gas below and above the critical temperature for the BCS phase transition are shown in Figs. 2(a) and 2(b), respectively. It is seen that when the transition occurs, a spatially broadened image appears. The physical situation is depicted in Fig. 3, where the small-scale structure induced by pairing causes light to scatter at a larger angle than that scattered by the cloud as a whole.

The normal signal is produced by coherent scattering and is, therefore, proportional to $(2 N)^{2}$, as can be verified by

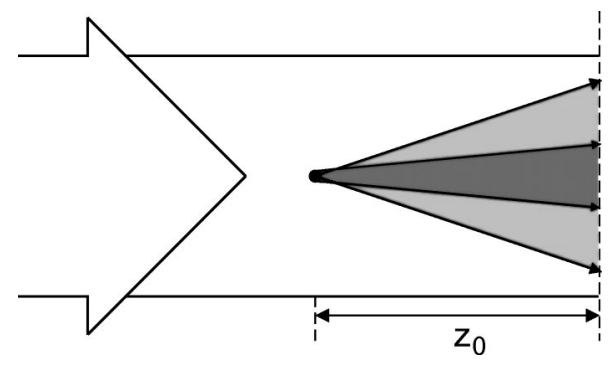

FIG. 3. Schematic of the imaging technique. The white area represents the incident probe laser, the dark gray represents the light coherently scattered by the cloud, and the light gray represents the light scattered by Cooper pairs. The small length scale of the pair structure scatters light at a relatively large angle, so by measuring the intensity in the far field, the components can be distinguished. Dark ground imaging techniques can be used to eliminate the contribution of the probe laser itself [12]. 
reference to Eq. (9). The pair signal, however, arises from inelastic scattering and is found using Eq. (10) to be proportional to the number of pairs $N_{p}$. This number is determined by the number of atoms in an energy shell of width $\Delta$ centered on $E_{F}$, so $N_{p} \approx 3 N \Delta / E_{F}$. For the parameters given above, $N_{p} \approx 3 \times 10^{4}$ and the ratio of the peak signal intensities is $I_{2}(0) / I_{1}(0) \approx 2 \times 10^{-7}$. Although it is difficult to experimentally measure a signal with such a large dynamic range, the pair signal can be revealed by using a nearly opaque spatial filter to attenuate the normal signal. If the diameter of the filter is chosen to be approximately equal to the spatial dimension of the normal signal image, it will affect only the central region of the pair signal, and both contributions can be observed with the same intensity scale.

Finally, we calculate the spectrum of the scattered light. For the normal degenerate ground state, a single spectral line is obtained at the frequency of the incident laser. For the superfluid state, the spectrum exhibits a double-peaked structure as shown in Fig. 4. The coherent peak is from scattering by the normal component. The frequency shift of the sideband is approximately twice the gap energy, indicating that the sideband arises from the formation of two quasiparticles. The long oscillating tail of the sideband is due to modulated broadening from the center-of-mass motion of atoms at the trap frequency. Hence, the presence of the shifted peak provides another effective method to detect the BCS phase transition and can be used to directly determine the gap energy.

The theory presented here was simplified by the neglect of spontaneous emission, permitting, for example, the assumption that $\Delta$ remains constant during probing. However, the pair signal depends on breaking pairs by inelastic scattering. The theory is, therefore, valid only in the weak-signal limit, where $\Gamma \ll T^{-1}$. Larger signals could be obtained experimentally by allowing $\Gamma \sim T^{-1}$, but quantitative interpretation would then be more difficult.

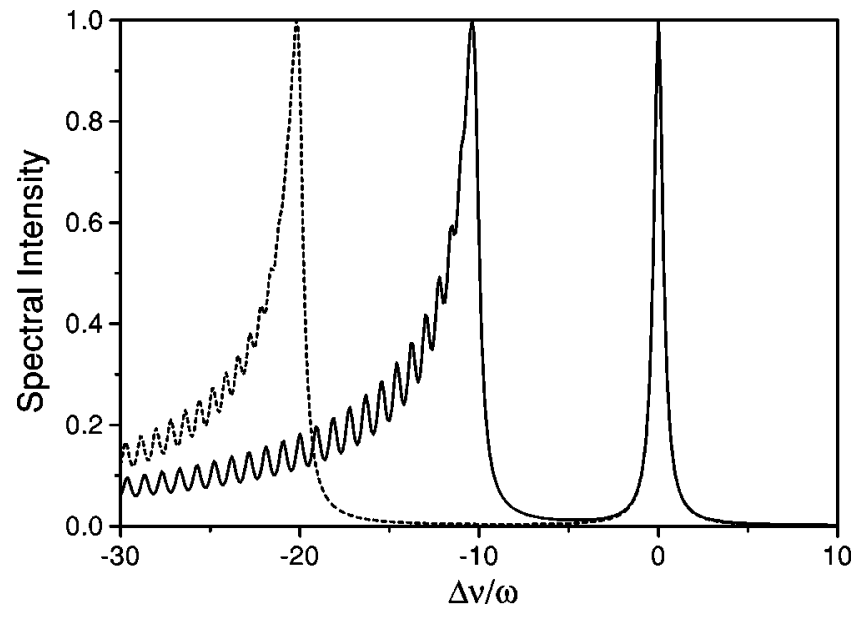

FIG. 4. The normalized scattered light spectrum of the scattered field. The frequency shift $\Delta \nu$ is scaled by the trap frequency $\omega$. For the solid curve, the gap energy $\Delta \approx 5 \hbar \omega=36 \mathrm{nK}$, and for the dotted curve, $\Delta=72 \mathrm{nK}$. A spatial filter with a transmission of $\sim 10^{-4}$ is used to reduce the strong signal from the normal component to the same level as that from the pair component. A small spontaneous scattering rate is assumed to give a width to the elastic peak.

In conclusion, we have studied off-resonance light scattering by a trapped degenerate Fermi gas. The results show that both spatial imaging and the scattered light spectrum give clear signatures for a BCS phase transition to a gaseous superfluid state.

The work in Australia was supported by the Australian Research Council, and a Macquarie University Research Grant. The work at Rice University was supported by the NSF, ONR, NASA, and the Welch Foundation. We are grateful to H.T.C. Stoof for helpful discussions. W.Z. thanks the atom-cooling group at Rice for their hospitality during his visit, and also thanks Karl-Peter Marzlin for his help.
[1] M. Anderson, J. R. Ensher, M. R. Matthews, C. E. Wieman, and E. A. Cornell, Science 269, 198 (1995); C. C. Bradley, C. A. Sackett, J. J. Tollett, and R. G. Hulet, Phys. Rev. Lett. 75, 1687 (1995); K. B. Davis, M.-O. Mewes, M. R. Andrews, N. J. van Druten, D. S. Durfee, D. M. Kurn, and W. Ketterle, ibid. 75, 3969 (1995).

[2] H. T. C. Stoof, M. Houbiers, C. A. Sackett, and R. G. Hulet, Phys. Rev. Lett. 76, 10 (1996); M. Houbiers, R. Ferwerda, H. T. C. Stoof, W. I. McAlexander, C. A. Sackett, and R. G. Hulet, Phys. Rev. A 56, 4864 (1997).

[3] J. Schneider and H. Wallis, Phys. Rev. A 57, 1253 (1998).

[4] G. Bruun, Y. Castin, R. Dum, and K. Burnett, e-print cond-mat/9810013; G. M. Bruun and K. Burnett, Phys. Rev. A 58, 2427 (1998).

[5] M. A. Baranov, Yu. Kagan, and M. Yu Kagan, Pis'ma Zh. Éksp. Teor. Fiz. 64, 273 (1996) [JETP Lett. 64, 301 (1996)].

[6] D. A. Butts and D. S. Rokhsar, Phys. Rev. A 55, 4346 (1997).

[7] A. J. Leggett, J. Phys. (France) 41, C7-19 (1980).
[8] E. R. I. Abraham, W. I. McAlexander, J. M. Gerton, R. G. Hulet, R. Côté, and A. Dalgarno, Phys. Rev. A 55, R3299 (1997).

[9] E. Tiesinga, B. J. Verhaar, and H. T. C. Stoof, Phys. Rev. A 47, 4114 (1993).

[10] Weiping Zhang, Phys. Lett. A 176, 225 (1993); Weiping Zhang, D. F. Walls, and Barry Sanders, Phys. Rev. Lett. 72, 60 (1994).

[11] G. Lenz, P. Meystre, and E. M. Wright, Phys. Rev. Lett. 71, 3271 (1993).

[12] J. Javanainen, Phys. Rev. Lett. 75, 1927 (1995); H. D. Politzer, Phys. Lett. A 209, 160 (1995).

[13] M. Born and E. Wolf, Principles of Optics, 6th ed. (Pergamon Press, Oxford, 1980); M. R. Andrews, M.-O. Mewes, N. J. van Druten, D. S. Durfee, D. M. Kurn, and W. Ketterle, Science 273, 84 (1996).

[14] B. Saleh, Photoelectron Statistics (Springer-Verlag, Berlin, 1978). 